\title{
Influence of Induced Convection on Transformation Kinetics During Rapid Solidification of Steel Alloys: The Retained Damage Model
}

\author{
DOUGLAS M. MATSON (D) ${ }^{1,2}$ \\ 1.-Mechanical Engineering Department, Tufts University, Medford, MA 02155, USA. \\ 2.—e-mail: matson@tufts.edu
}

\begin{abstract}
The Retained Damage Model successfully predicts the incubation delay during transformation from ferrite to austenite in the presence of an applied external flow field during rapid solidification of ternary stainless-steel alloys. The model incorporates two new features-conservation of the free energy associated with undercooling of the primary metastable phase, and use of a modified Read-Shockley approach to quantify defect energy induced by melt shear. Healing of the microstructure could reduce the amount of free energy retained, but, for the alloys considered in this work, it was found that the model is not sensitive to this phenomenon, and thus $100 \%$ of available free energy is retained to provide an additional transformation driving force, significantly shortening the incubation period. Use of a dimensionless approach allows comparison between systems with very different thermophysical properties, and highlights the similarity in response to local flow conditions over a wide range of compositions.
\end{abstract}

\section{INTRODUCTION}

Electromagnetic stirring has been successfully used for many decades to improve product quality during continuous casting operations. ${ }^{1}$ One approach to investigating how convection influences metastable phase solidification, with subsequent transformation to a stable phase in steel alloys, is to utilize containerless levitation processing. Electromagnetic levitation (EML) leads to significant convection when compared to electrostatic levitation (ESL). ${ }^{2,3}$ Conducting EML experiments in space allows for investigation of a wide range of induced convection because the lack of gravity means levitation forces can be significantly reduced, ${ }^{4}$ with a concomitant reduction in both applied electromagnetic field strength and induced convection. In each of these approaches, magnetohydrodynamic modeling (MHD) is used to predict the level of convection and the rate of shear in the undercooled liquid during processing. ${ }^{5-7}$

(Received June 16, 2020; accepted September 2, 2020; published online September 22, 2020)
The hypo-eutectic FeCrNi stainless-steel alloy family solidifies in a two-step process known as double recalescence. ${ }^{8}$ The temperature profile exhibits a first rise during the formation of metastable bcc-ferrite from the undercooled liquid, followed by an almost immediate second rise during the formation of the stable fcc-austenite within the pre-existing metastable semi-solid. Because these alloys do not significantly partition, the second temperature rise associated with the transformation to the stable phase, $\Delta T_{\mathrm{s}}$, is the difference between the metastable phase liquidus, $T_{\mathrm{L}, \mathrm{m}}$, and stable phase liquidus, $T_{\mathrm{L}, \mathrm{s}}$, as defined by the metastable extension of phase boundaries from the equilibrium phase diagram: ${ }^{9}$

$$
\Delta T_{\mathrm{s}}=T_{\mathrm{L}, \mathrm{s}}-T_{\mathrm{L}, \mathrm{m}}
$$

No matter what the primary undercooling was during the formation of the metastable phase, the thermodynamic driving force, $\Delta G_{\mathrm{s}}$, associated with the subsequent transformation from metastable bcc to stable fcc should be constant, according to classical nucleation theory (CNT), ${ }^{10,11}$ since the 
volume free energy change is only a function of the heat of fusion, $\Delta H_{\mathrm{s}}$, the melting point, $T_{\mathrm{L}, \mathrm{s}}$, and the undercooling, $\Delta T_{\mathrm{s}}$.

If the driving force is constant, then it would be expected that the incubation period between the two recalescence events, known as the delay time, should be constant. However, the observed delay is a weak function of primary undercooling and a strong function of melt convection, ${ }^{12}$ and, since the delay is not independent of both, a new model needed to be developed.

Quantification of the incubation period is accomplished based on the principle of microscopic reversibility, ${ }^{13}$ where clusters grow reversibly at the rate that they would decompose. Since only one cluster is required for initiation of the transformation, the fastest nucleus to form anywhere in the material will result in complete conversion to the stable phase. The delay, $\tau$, is a function of transformation temperature, $T$, the attachment rate, $\beta$, and the curvature of the free energy, $G$, with respect to an incremental addition of an atom to a cluster at its critical size. The mechanism dependent constant, $\kappa$, is typically set to a value of 4 for grain boundary nucleation. ${ }^{14}$ Note that the negative sign is included because the curvature is negative:

$$
\tau=-\frac{\kappa k_{B} T}{\beta\left(\frac{\partial^{2} \Delta G}{\partial n^{2}}\right)}
$$

Mathematical evaluation of the curvature requires assumptions on cluster geometry, and previous work has shown that nucleation of the stable phase occurs along sub-grain boundaries within the metastable solid, and having a geometry characterized as adjoining dual hemispherical caps. $^{8,15,16}$ By eliminating the cluster critical radius using CNT, the curvature may be evaluated, as shown in Eq. 3. Substituting this result into Eq. 2 produces a relationship where the delay, $\tau$, becomes inversely proportional to the free energy change, $\Delta G$, raised to a characteristic exponent which is geometry- and mechanism-dependent. ${ }^{17-19}$ Evaluation of experimental data indicates that the best fit for $\mathrm{FeCrNi}$ is obtained when the attachment rate, $\beta$, is constant with time such that this exponent becomes a value of four, ${ }^{20}$ as shown in Eq. 4; these results are functionally consistent with the work proposed by Kantrowicz ${ }^{21}$ and Kashchiev: ${ }^{22}$

$$
\begin{gathered}
\left.\frac{\partial^{2} \Delta G}{\partial n^{2}}\right|_{r *}=-\frac{\Delta G_{T}^{4}}{32 \pi \gamma^{3} f(\theta)}\left(\frac{\Omega}{N_{\mathrm{A}}}\right)^{2} \\
\tau=\frac{128 \pi k_{\mathrm{B}} T \gamma^{3} f(\theta)}{\beta \Delta G_{T}^{4}}\left(\frac{N_{\mathrm{A}}}{\Omega}\right)^{2}
\end{gathered}
$$

The expression includes alloy-specific quantities, such as terms describing surface energy, $\gamma$, molar volume, $\Omega$, and wetting angle, $\theta$, and physical constants, such as the Boltzmann constant, $k_{\mathrm{B}}$, and Avogadro's number, $N_{\mathrm{A}}$.

The purpose of this paper is to present the implications of the new Retained Damage Model (RDM) in more detail as a follow-on to a previous JOM paper, ${ }^{23}$ which described how selection of processing parameters during testing allows for control of convection during rapid solidification. The current paper presents background material on the assumptions dealing with the selection of nucleation model geometry and the mechanism controlling the cluster development, and then examines experimental measurements of undercooling and delay time to evaluate how the results conform to model predictions. Next, an evaluation of the sensitivity of model prediction is performed to evaluate the impact of any adjustable parameters. Finally, the model is applied to the family of ternary FeCrNi alloys which show double recalescence during rapid solidification in EM-induced fluid flow fields.

\section{EXPERIMENTAL}

\section{Containerless Processing}

Levitation processing of reactive molten metal alloy samples eliminates crucible-induced contamination; typically, the sample temperature is monitored using optical pyrometry, while solidification is imaged using high-speed digital cinematography during free cooling. When experiments target quiescent melt conditions, ESL is used whereby the sample is positioned between two charged plates in a vacuum and heated by a laser. ${ }^{24}$ When significant convection is desired, terrestrial EML is selected because the magnetic field required to levitate the sample induces significant flow. ${ }^{25}$ By conducting tests using EML in microgravity, the convection can be controlled between these extremes, since both levitation forces and induced convection is reduced. $^{26}$ For FeCrNi testing using either EML technique, sample evaporation was minimized by conducting experiments using an inert shieldinggas environment.

\section{Alloy Selection}

Samples were fabricated by arc-melting $5 \mathrm{~N}$ pure elements in the appropriate quantities to obtain the desired ternary composition. Space samples had a composition, in weight percent, of $60 \% \mathrm{Fe}, 20 \% \mathrm{Cr}$ and $20 \% \mathrm{Ni}$. Terrestrial samples included the space alloy as well as a range of compositions from the $72 \% \mathrm{Fe}$ isopleth, with compositions of $11 \%, 12 \%$, 
Table I. Properties specific to the FeCrNi alloy family

\begin{tabular}{|c|c|c|c|c|c|}
\hline Property & Units & 20-20 alloy & 11-17 alloy & 12-16 alloy & 14-14 alloy \\
\hline$C_{\mathrm{Ni}}$ & $(\mathrm{wt} \% \mathrm{Ni})$ & 20 & 17 & 16 & 14 \\
\hline$\Delta T_{\mathrm{s}}$ & $(\mathrm{K})$ & 44.2 & 46.3 & 39.2 & 23.9 \\
\hline$T_{\mathrm{L}, \mathrm{s}}$ & (K) & 1713.0 & 1744.3 & 1743.1 & 1739.5 \\
\hline$T_{\mathrm{L}, \mathrm{m}}$ & (K) & 1668.8 & 1698.0 & 1703.8 & 1715.6 \\
\hline$\Delta H_{\mathrm{s}}$ & $(\mathrm{J} / \mathrm{mol})$ & 11,235 & 12,319 & 12,152 & 11,855 \\
\hline$\Delta H_{\mathrm{m}}$ & $(\mathrm{J} / \mathrm{mol})$ & 10,629 & 11,226 & 11,009 & 10,589 \\
\hline$N_{\Xi}$ & - & 0.9549 & 0.9189 & 0.9103 & 0.8921 \\
\hline$\Delta G_{\mathrm{R}}$ & $\left(\mathrm{J} / \mathrm{m}^{3}\right)$ & $3.87 \times 10^{7}$ & $4.36 \times 10^{7}$ & $3.65 \times 10^{7}$ & $2.17 \times 10^{7}$ \\
\hline$\tau_{\mathrm{R}}$ & (s) & 0.247 & 0.153 & 0.314 & 2.501 \\
\hline
\end{tabular}

and $14 \% \mathrm{Cr}$. In the remainder of the paper, these compositions will be referenced by $\mathrm{Cr}-\mathrm{Ni}$ content such that investigations were conducted on 20-20, 11-17, 12-16, and 14-14 alloys. Table I contains a summary of the key properties used to perform the transformation calculations. Metastable and stable properties for the various alloys were obtained using the commercially available computational thermodynamics program ThermoCalc v.2017b with the FEDEMO database.

\section{RETAINED DAMAGE MODEL (RDM)}

\section{Approach}

The RDM is based on the premise that, since undercooling and convection result in a reduction in the incubation period, there must be additional free energy contained within the microstructure of the metastable phase to drive the transformation. The inspiration behind this approach is the realization that this type of process is commonly associated with cold working of a metal. Damage energy introduced into the crystal structure results in defects which can be healed during either recrystallization or subsequent phase transformation. The concentration of these defects increases as the growth rate of the metastable phase increases with primary undercooling, and it increases with an enhancement of melt shear due to the flow field.

In the case of ferrous alloys, the physical interpretation involves the formation of spherical cap clusters leading to secondary nucleation along subgrain boundaries, which are associated with the alignment of a dislocation network in the form of a low-angle tilt boundary. Grain boundary energies were evaluated as a function of the tilt angle, and thus dislocation concentration, by Yasuda ${ }^{27}$ using atomistic simulation, and subsequently employed to define the critical misorientation, allowing the defect structure to serve as a potent heterogeneous nucleation site. ${ }^{16}$

It is difficult to track the role of specific defect types, and thus a global approach is indicated such that the sum of the energy contributions from each imperfection classification is manifested as damagefree energy. When evaluating the retained energy due to primary undercooling, it is unclear how to quantify the magnitude of the contribution, but a clear limit is imposed by the thermodynamics of the solid-liquid transformation-there can be no more energy retained than was originally present to drive primary solidification, $\Delta G_{\mathrm{m}}$. When evaluating the retained energy due to the interaction between the metastable solid and melt shear, $\Delta G_{\mathrm{c}}$, it is obvious that the dislocation concentration, and the associated stress, become key factors. The magnitude of the contribution can be estimated using a modification of the Read-Shockley equation, ${ }^{28}$ where the boundary tilt angle, and thus the dislocation concentration, is assumed to be proportional to the applied melt shear, $\dot{\gamma}$. Mathematical manipulation yields the relationship shown in the third part of Eq. 5. ${ }^{19}$ The constants in this equation are empirically determined from experimental data from a plot of the dependent variable, $\Delta G_{c} / \dot{\gamma}$, as a function of the independent variable, $\ln (\dot{\gamma})$, which yields a straight line with a negative slope of $\Delta_{m}$ and an intercept, $\Delta_{b}$.

In the RDM, there are three components to the volume free energy term, $\Delta G_{\mathrm{T}}$, used in conjunction with CNT. These are (1) CNT free energy associated with the transformation from primary bcc to stable fcc $\Delta G_{\mathrm{s}}$, (2) retained damage energy associated with undercooling of the metastable bcc phase, $\Delta G_{\mathrm{m}}$, and (3) retained damage energy associated with the convection field which existed during primary phase growth, $\Delta G_{\mathrm{c}}$. Thus, the total free energy driving the transformation is the sum of:

$$
\begin{aligned}
\Delta G_{\mathrm{T}} & =\Delta G_{\mathrm{s}}+\Delta G_{\mathrm{m}}+\Delta G_{\mathrm{c}} \text { for } \Delta G_{\mathrm{s}}=\frac{\Delta H_{\mathrm{s}} \Delta T_{\mathrm{s}}}{T_{\mathrm{L}, \mathrm{s}}} \\
\Delta G_{\mathrm{m}} & =f_{x} \frac{\Delta H_{\mathrm{m}} \Delta T_{\mathrm{m}}}{T_{\mathrm{L}, \mathrm{m}}} \\
\Delta G_{\mathrm{c}} & =f_{x} \Delta_{m} \dot{\gamma}\left[\Delta_{b} / \Delta_{m}-\ln \gamma\right]
\end{aligned}
$$

In this treatment, the undercooling, $\Delta T_{\mathrm{s}}$, is defined in Eq. 1 , and $\Delta T_{\mathrm{m}}$ is the undercooling of the liquid relative to the metastable phase liquidus. The first term represents the volume free energy associated with CNT. The second term represents the damage introduced during primary phase 
solidification. The third term represents the contribution to the retained free energy from the interaction between the growing metastable phase and the convective field throughout primary recalescence. Because healing of the primary phase prior to secondary recalescence may occur, the second and third terms are modified by the inclusion of an adjustable retained energy parameter, $f_{x}$, to track the fraction of available free energy that is retained.

Note that the additional contribution by the retained free energy terms creates conditions that promote nucleation but do not impact the driving force for subsequent growth. Stable phase growth into the semi-solid mixture of the metastable solid and the remaining liquid is independent of both primary undercooling and melt shear; the growth rate is controlled by $\Delta T_{\mathrm{s}}$ from the phase diagram, as described in Eq. 1, and the enthalpy of the transformation. This apparent paradox is resolved by highlighting the distinction between free energydriven nucleation and thermally-driven growth. ${ }^{9,29-31}$

\section{Assessment}

Double recalescence is only possible if the liquid sample is undercooled to an extent such that the temperature drops below the liquidus of the metastable phase. Under these conditions, $\Delta T_{\mathrm{m}}=$ 0 , and this minimum undercooling is equivalent to $\Delta T_{\mathrm{s}}$, as defined in Eq. 1, and serves as a baseline for defining the reference conditions used in developing two key dimensionless quantities. Note that the reference values represent the minimum undercooling and the maximum delay time for any given alloy system. Since it is experimentally difficult to evaluate behavior under these conditions, the values used must be extrapolated from test results. This is functionally equivalent to assigning an appropriate $\beta$ to characterize the system attachment flux, based on an empirically evaluated constant cluster attachment success rate. ${ }^{19}$

The two dimensionless groups that are used are independently identified by the two measurable quantities, the undercooling and the delay time. The dimensionless free energy parameter, $N_{M}$, is defined as the ratio of the total free energy, $\Delta G_{\mathrm{T}}$, (which in turn is a function of observed undercooling) to the baseline reference minimum undercooling defined by the phase diagram, $\Delta G_{\mathrm{R}}$. This reference condition is mathematically equivalent to $\Delta G_{\mathrm{s}}$, as defined in Eq. 5. The dimensionless delay parameter, $N_{\tau}$, is defined as the ratio of the observed experimental delay, $\tau_{\mathrm{EXP}}$, to the reference maximum delay time, $\tau_{\mathrm{R}}$.

Experimental data can then be used to evaluate how well the theory predicts behavior. One key parameter is the definition of the reference maximum delay time. This is accomplished by plotting the ESL data and extrapolating the delay to the minimum system undercooling, $\Delta T_{\mathrm{s}}$, from the phase diagram. Next, the ESL data are used to evaluate the retained energy parameter, $f_{x}$. ESL testing involves conditions where induced convection approaches zero such that the third term in Eq. 5 goes to zero. Since the first term is known from the phase diagram, only the second term remains as an unknown. To accomplish this analysis, the observed delay time at a given undercooling is inserted into Eq. 4 to define how much total free energy is predicted to be required. The value of $f_{x}$ is obtained by comparing this value to a calculation of the actual free energy supplied based on the primary undercooling. At the extreme, if $f_{x}=0$, then the material will have fully recrystallized and no free energy is retained. Previous work ${ }^{20}$ indicates that $f_{x} \rightarrow 1$, such that $100 \%$ of the available free energy from primary recalescence is retained within the metastable microstructure and available to drive the subsequent transformation.

Finally, experimental data from EML testing are used to define the contribution to retained damagefree energy due to melt shear induced by the convective flow field. For these tests, the first two terms are now known and only the third remains to be identified using techniques similar to those described above. The observed delay is used to predict the required total free energy, and this value is plotted to obtain the empirical constants in the modified Read-Shockley equation. With knowledge of these constants, any test condition with known undercooling and known melt shear may be simulated.

\section{RESULTS AND DISCUSSION}

\section{Model Sensitivity to Adjustable Parameter Selection}

The first decision that must be made is to define both the system geometry ${ }^{15}$ and the mechanism controlling the transformation kinetics. The general form of the relationship between delay and free energy is that $\tau$ is proportional to $\Delta G_{\mathrm{T}}^{-m}$, where the mechanism exponent, $m$, varies from 2 to $4 .^{20}$ Figure 1 contains a plot of model predictions for two potential mechanisms involving either the diffusion of atoms through the parent metastable phase, $D_{\text {bulk }}$, such that the exponent is $m=2$ (consistent with Shao and Tsakiropoulos ${ }^{17}$ ), or having a constant attachment rate, $\beta$, such that the exponent is $m=4$ (consistent with Kantrow$i^{21}{ }^{21}$. Clearly, the behavior is best described by the constant attachment rate mechanism for both the quiescent ESL test results with no induced flow and for the turbulent EML test results with application of a significant induced convective flow field.

Once the mechanism is identified, the next step is to define the attachment parameter, $\beta$. This is an adjustable parameter that quantifies the success rate for incremental growth of the cluster. The sensitivity to changes in assumed values of $\beta$ is quite large, as shown in Fig. 2, where curves 
representing predictions based on assumed values of $2 \times 10^{7} \leq \beta \leq 8 \times 10^{7}$ (small dotted lines and large dotted lines, respectively) such that $0.5 \geq \tau_{R} \geq$ 0.1 based on the inversely proportional relationship presented in Eq. 4. Recursive optimization shows that the best fit is obtained for $\beta=4 \times 10^{7}$ atoms $/ \mathrm{s}$ (solid line), corresponding to a reference delay time value of $\tau_{\mathrm{R}}=0.247 \mathrm{~s}$ for the 20-20 alloy. This condition is indicated by the solid red star in Fig. 2, and is tabulated in Table I.

In order to evaluate the empirical modified ReadShockley parameters, the variable, $\Delta G_{\mathrm{c}} / \dot{\gamma}$, is plotted as a function of $\ln (\dot{\gamma})$, as seen in Fig. 3, and linear regression yields values for the intercept $\Delta_{b}=1.69$ $\pm 0.009 \times 10^{6} \mathrm{Js} / \mathrm{m}^{2}$ and a negative slope, such that $\Delta_{m}=2.52 \pm 0.012 \times 10^{5} \mathrm{Js} / \mathrm{m}^{2}$ These values are used to predict the influence of convective melt shear due to imposed flow field on the incubation delay.

Finally, the impact of assuming a value of the retained energy parameter, $f_{x}$, is checked. As seen in Fig. 4, there is no significant impact on model performance based on selection of any appropriate value over the entire range for the retained energy parameter, which justifies adoption of the mathematical simplification where $f_{x} \rightarrow 1$, implying that all the damage energy is retained during the incubation period and the energy associated with these defects, and their associated stress, remains available to augment nucleation of the stable phase. This, therefore, ceases to be considered as an adjustable parameter.

The consequence is that damage significantly reduces the delay time, and that there is only one adjustable parameter required for matching the model predictions to the experimental results. Once a mechanism is defined, the identification of the delay time reference condition depends solely on the selection of the attachment rate term.

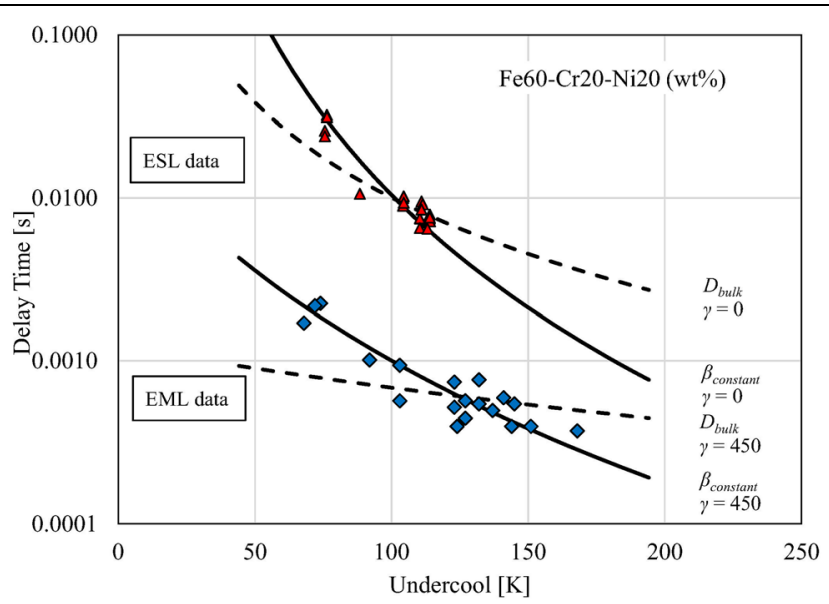

Fig. 1. Selection of the mechanism controlling transformation kinetics for the 20-20 steel alloy.

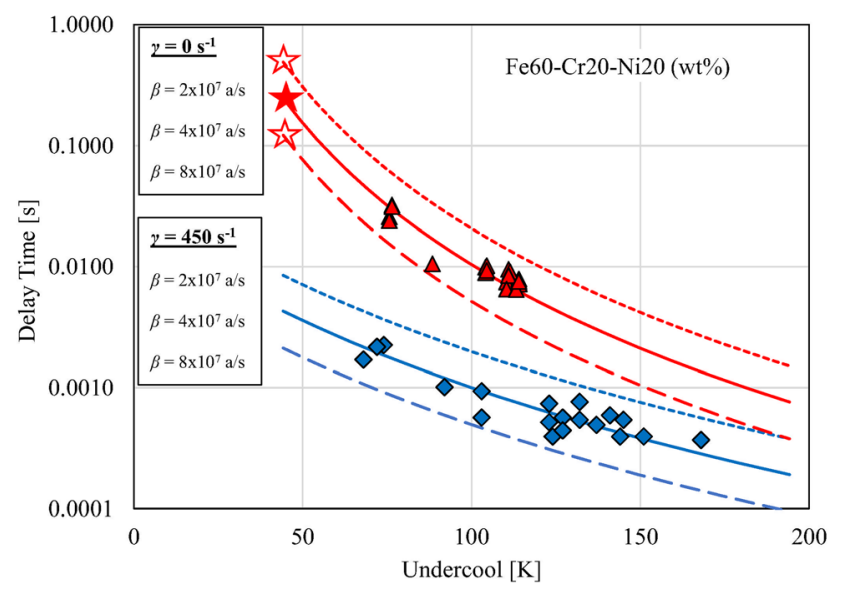

Fig. 2. Influence of selection of an attachment rate on model predictions for the reference delay time.

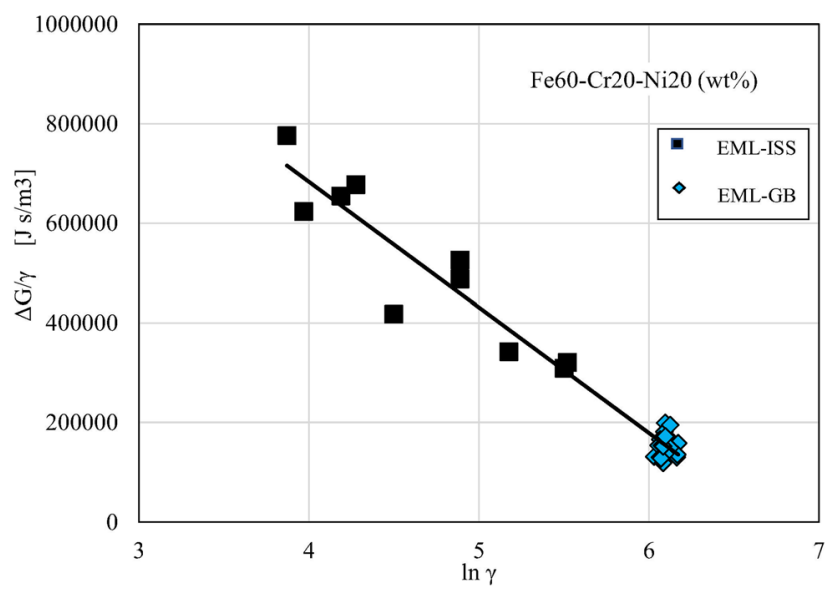

Fig. 3. Empirical evaluation of the modified Read-Shockley parameters from high-shear EML data.

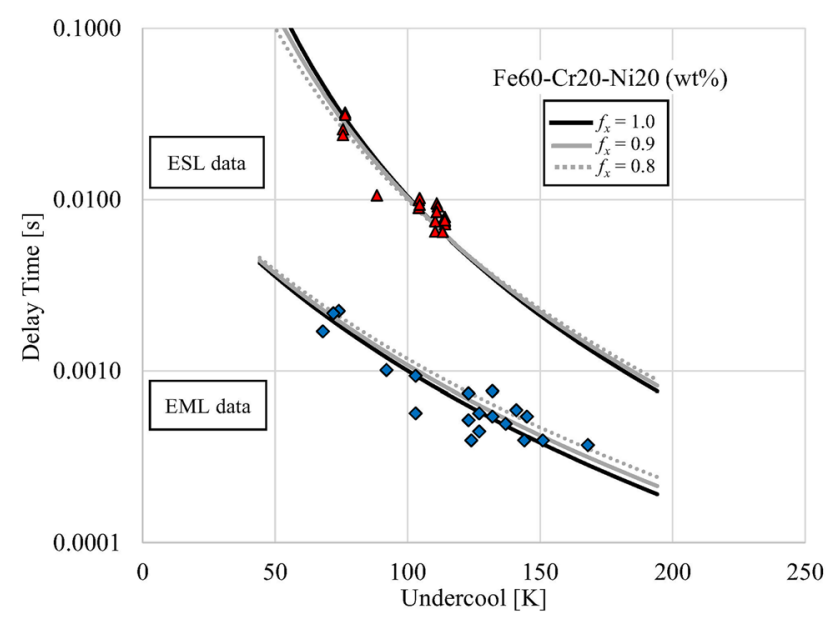

Fig. 4. Influence of the adjustable parameter used to track healing of the damaged microstructure. 


\section{Dimensionless Approach Applied to Other Alloys Within the Ternary FeCrNi System}

Table I shows the range of values for key temperatures, enthalpies, and reference quantities for the various $\mathrm{FeCrNi}$ compositions investigated. These properties can be manipulated to identify a third dimensionless group characterizing the metastable to stable phase transformation:

$$
\begin{gathered}
N_{\Xi}=\frac{\Delta H_{\mathrm{M}} T_{\mathrm{M}} / \Omega_{\mathrm{M}}}{\Delta H_{\mathrm{S}} T_{\mathrm{S}} / \Omega_{\mathrm{S}}} \quad \text { such that } \\
\frac{\Delta G_{\mathrm{T}}}{\Delta G_{\mathrm{R}}}=1+\left(\frac{\Delta T}{\Delta T_{\mathrm{s}}}-1\right) N_{\Xi}
\end{gathered}
$$

If the properties of the metastable phase are unknown, we can approximate behavior by assuming that the metastable and stable phase properties are similar enough to assume $N_{\Xi} \rightarrow 1$, such that the value of the dimensionless free energy parameter becomes a ratio of undercoolings instead of free energies:

$$
N_{\mathrm{M}}=\frac{\Delta G_{\mathrm{T}}}{\Delta G_{\mathrm{R}}} \rightarrow \frac{\Delta T}{\Delta T_{\mathrm{s}}}
$$

Luckily, in the FeCrNi system, computational thermodynamic predictions are available, as set out in Table I. Values of $N_{\Xi}$ vary significantly with the composition for this system, leading to significant differences in $\Delta G_{\mathrm{R}}$ and $\tau_{\mathrm{R}}$. Of particular importance is the variation in the temperature rise during the transformation, $\Delta T_{\mathrm{s}}$, as defined by the phase diagram. This dramatically affects the calculation of the value for the reference delay time. The reference thermodynamic driving force, $\Delta G_{\mathrm{R}}$, is only a weak function of composition, with differences mainly due to alloy-specific variation in this rise. Values common to all the compositions within the family are the metastable surface energies, $\gamma_{\mathrm{M}}=0.75 \mathrm{~J} / \mathrm{m}^{216}$ and $\gamma_{\mathrm{S}}=0.40 \mathrm{~J} / \mathrm{m}^{2}$, the resulting wetting angle, $\theta=20.4^{\circ}{ }^{19}$ and the molar volume, $\Omega=7.5 \times 10^{-6} \mathrm{~m}^{3} / \mathrm{mol}^{19}$ We will also assume that the retained energy fraction, $f_{x}=1 .^{20}$

It would be desirable to know the compositional dependence of the one adjustable parameter, the attachment rate, $\beta$, but there is insufficient data at low melt shear to evaluate this parameter for the $72 \mathrm{wt} \%$ isopleth. To overcome this challenge, we will assume that $\beta=4 \times 10^{7}$ atoms/s as defined by the 20-20 composition. With this assumption, the prediction of the reference delay becomes possible using Eq. 4. Values range from $0.15 \mathrm{~s}$ to $2.50 \mathrm{~s}$ for $\tau_{\mathrm{R}}$.

It would also be desirable to know the compositional dependence of the Read-Shockley parameters, but, to do this, we would need microgravity data which are currently unavailable. Again, we will assume that, for these quantities, there is only a weak dependence with composition, and so use the values identified for the $60 \mathrm{wt} \% \mathrm{Fe}$ isopleth and apply them to the $72 \mathrm{wt} \%$ isopleth.
Experimental data is presented in Fig. 5 for comparison with the dimensionless presentation in Fig. 6.

In the figure, the diagonal represents the theoretical negative slope corresponding to an exponent of $m=4$ based on Eq. 4. Regression of the experimental data shows that the 20-20 alloy exhibits a negative slope of $3.992 \pm 0.027$, while the family shows negative $4.013 \pm 0.023$, despite having to assume negligible compositional dependence for both the attachment rate and the modified ReadShockley parameters.

It should be emphasized that the two dimensionless numbers used in Fig. 6 are developed from independent experimentally measured quantities. In the case of the dimensionless delay time, $N_{\tau}$, the measured delay time during a specific test is divided by the reference value representing the longest possible incubation period for the system:

$$
\begin{gathered}
N_{\tau}=\frac{\tau_{\mathrm{EXP}}}{\tau_{\mathrm{R}}} \\
N_{\mathrm{M}}=\frac{\Delta G_{\mathrm{T}}}{\Delta G_{\mathrm{R}}}
\end{gathered}
$$

In contrast, the dimensionless driving force is based on the undercooling of a specific test and various thermophysical properties-the key to which is the minimum undercooling, $\Delta T_{\mathrm{s}}$, from the phase diagram. The reference condition for these two parameters is shown with a solid red star in Fig. 2.

\section{Future Work}

The predictive capability of the model when extending the results from the $20-20$ alloy to alloys along a different $\mathrm{Fe}$ isopleth was unexpected. Measuring the modified Read-Shockley empirical constants for other alloys is an obvious next step, but, in order to accomplish this under the current

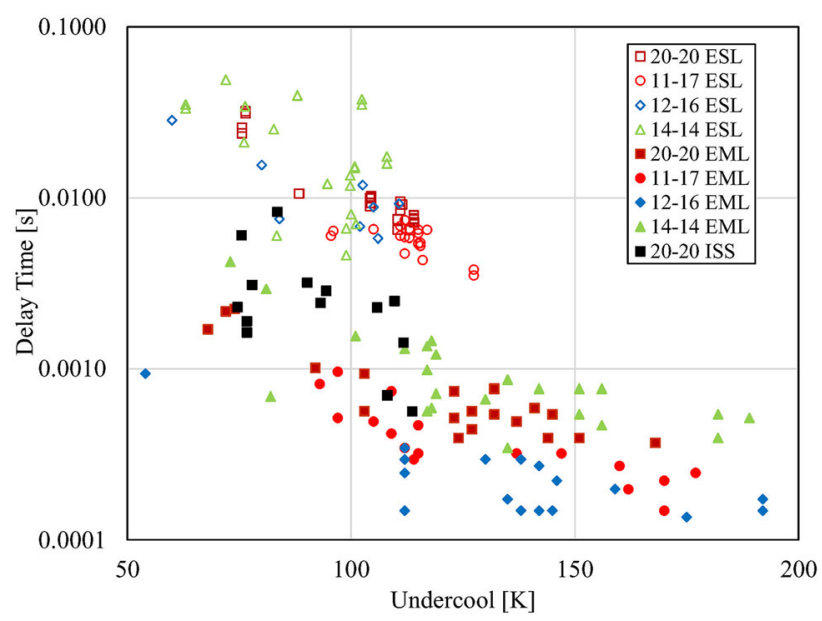

Fig. 5. Experimentally observed delay times plotted as a function of temperature for the $\mathrm{FeCrNi}$ family. 


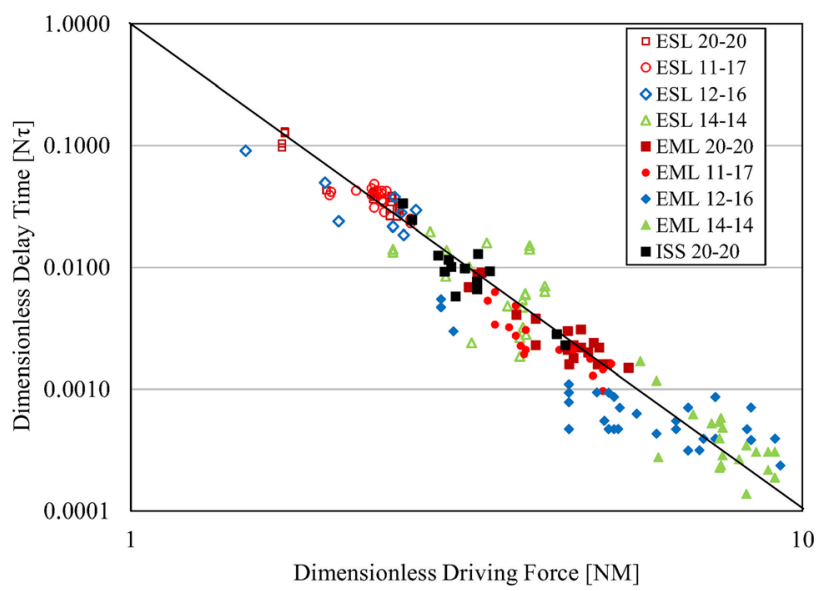

Fig. 6. Dimensionless evaluation of experimental results for delay as a function of thermodynamic driving force using the Retained Damage Model approach for the FeCrNi family.

approach, it is required that additional space testing be conducted. These experiments are currently in the planning stage, and it is hoped that results will become available in the near future.

Although the model performs well, physical evidence is lacking. It is hoped that testing can be accomplished to obtain experimental verification that the retained defect structure is enhanced by undercooling and by convection. This is difficult in the $\mathrm{FeCrNi}$ system, since the delay times are quite short and quenching experiments are not effective. The transformation completely obliterates any metastable structures, because the melting point of ferrite is significantly lower than stable austenite, such that, during the transformation, any metastable solid is rapidly melted. ${ }^{29}$ Preliminary efforts to evaluate the dislocation density as a function of processing condition using x-ray diffractometry were inconclusive, due to the limits imposed by the synchrotron data acquisition systems. $^{23}$

Identifying a different system with more sluggish transformation kinetics is desirable. Initial tests on $\mathrm{FeCo}$ alloys show promise, but investigations on TiAl alloys show healing to be significant as delay times get longer. Extension of the model to other non-ferrous alloy systems should give insight into the balance between retained damage and healing processes.

\section{CONCLUSION}

The incubation delay during transformation from a metastable ferrite to a stable austenite in $\mathrm{FeCrNi}$ alloys depends on the undercooling and on the melt shear due to convective flow during rapid solidification. A global thermodynamic approach is embraced; the exact form of the defect structure which embodies this damage is not identified but rather the energy that is retained within the metastable solid is summed, in order to estimate how additional energy becomes available to drive the transformation in a manner similar to cold working. As in cold working, healing may reduce the available driving force, but for these alloys this is not observed, and thus the fraction of energy which is retained is $f_{x}=1$.

The Retained Damage Model extends existing models by incorporating terms based on the conservation of the free energy driving primary recalescence and the damage induced by flow. A modified Read-Shockley approach is utilized, and a method is developed to estimate the empirical parameters relating melt shear and the damage energy retained. Compositional variation in these parameters, and in the attachment rate governing cluster growth, are found to not be significant. The attachment rate is determined to be a constant value of $\beta=4 \times 10^{7}$ atoms $/ \mathrm{s}$.

In order to be able to compare the behavior of alloys across a wide compositional range involving broad changes in specific thermophysical properties, a dimensionless approach was developed. The dimensionless delay time and dimensionless driving force were defined based on two independent measurements, the delay and the undercooling for a specific test. To make these values dimensionless, a reference condition was identified representing the maximum delay possible at the minimum undercooling that is achievable as defined by the phase diagram. When the FeCrNi alloys are plotted using this approach, a linear relationship emerges with a slope consistent with the theoretical value of -4 .

\section{ACKNOWLEDGEMENTS}

The authors acknowledge collaborative support by team members from the Microgravity User Support Center (MUSC) through access to the ISS-EML facility, which is a joint undertaking of the European Space Agency (ESA) and the German Aerospace Administration (DLR). This project was sponsored by NASA under Grants NNX17AH41G and 80NSSC19K0256. The author also acknowledges the support from the ESA and the DLR administration, as this work was conducted under the collaborative framework of ThermoLab AO-2009-1020.

\section{REFERENCES}

1. A.A. Tzavaras and H.D. Brody, JOM 36, 31 (1984).

2. R.W. Hyers, D.M. Matson, K.F. Kelton, and J.R. Rogers, Ann. N. Y. Acad. Sci. 1027, 474 (2004).

3. D.M. Matson, D.J. Fair, R.W. Hyers, and J.R. Rogers, Ann. N. Y. Acad. Sci. 1027, 435 (2004).

4. D.M. Matson, R.W. Hyers, T. Volkmann, and H.-J. Fecht, J. Phys: Conf. Ser. 327, 1 (2011).

5. X. Xiao, J. Lee, R.W. Hyers, and D.M. Matson, npj Microgravity 5,7 (2019).

6. X. Xiao, R.W. Hyers, and D.M. Matson, Int. J. Heat Mass Transf. 136, 532 (2019).

7. E.B. Baker, J. Nawer, X. Xiao, and D.M. Matson, npj Microgravity 6, 9 (2020).

8. T. Koseki and M.C. Flemings, Met. Trans. 26A, 2991 (1995).

9. D.M. Matson and R.W. Hyers, Philos. Mag. 86, 3795 (2006).

10. D. Turnbull, J. Appl. Phys. 21, 1022 (1950).

11. J.W. Christian, The Theory of Transformation in Metals and Alloys (Oxford: Pergamon, 1975), p. 418. 
12. D.M. Matson, R.W. Hyers, and T. Volkmann, J. Jpn. Soc. Microgravity Appl. 27, 238 (2010).

13. J. Feder, K.C. Russell, J. Lothe, and G.M. Pound, Adv. Phys. 15, 111 (1966).

14. K.C. Russell, Acta Met. 17, 1123 (1969).

15. D.M. Matson, Solidification of Containerless Undercooled Melts, ed. D.M. Herlach and D.M. Matson (New York: Wiley, 2012), p. 231.

16. D.M. Matson and X. Xiao, Mater. Lett. 212, 256 (2018).

17. G. Shao and P. Tsakiropoulos, Acta Metall. Mater. 49, 2937 (1994).

18. C. Yang, F. Liu, G. Yang, Y. Chen, N. Liu, J. Li, and Y. Zhou, Mat. Sci. Engr. A 458, 1 (2007).

19. D.M. Matson, npj Microgravity 4, 22 (2018).

20. D.M. Matson, X. Xiao, and S. Jeon, IOP Conf. Ser. Mat. Sci. Eng. 529, 012012 (2019).

21. A. Kantrowitz, J. Chem. Phys. 19, 1097 (1951).

22. D. Kashchiev, Surf. Sci. 18, 389 (1969).

23. D.M. Matson, X. Xiao, J.E. Rodriguez, J. Lee, R.W. Hyers, O. Shuleshova, I. Kaban, S. Schneider, C. Karrasch, S. Burggraff, R. Wunderlich, and H.-J. Fecht, JOM 69, 1311 (2017).
24. J. Nawer, X. Xiao, M.P. Sansoucie, and D.M. Matson, High Temp. High Press. 49, 17 (2020).

25. L.M. Kensel, The Influence of Convection on Nucleation Phenomena During Double Recalescence. Masters Thesis, (MIT, 2002).

26. J. Nawer, S. Gossé, and D.M. Matson, JOM 72, 3132 (2020).

27. M. Yoshiya, K. Nakajima, M. Watanabe, N. Ueshima, T. Nagira, and H. Yasuda, Mater. Trans. 56, 1461 (2015).

28. W.T. Read and W. Shockley, Phys. Rev. 78, 275 (1950).

29. J.E. Rodriguez and D.M. Matson, Acta Mater. 129, 408 (2017).

30. D.M. Matson, W. Löser, and M.C. Flemings, Solidification 1999, ed. W.H. Hofmeister, J.R. Rogers, N.B. Singh, S.P. Marsh, and P.W. Voorhees (Warrendale: TMS, 1999), p. 99

31. J. Rodriguez, C. Kreischer, T. Volkmann, and D.M. Matson, Acta Mater. 122, 431 (2017).

Publisher's Note Springer Nature remains neutral with regard to jurisdictional claims in published maps and institutional affiliations. 